\title{
A Matrix Approach to Hypergraph Stable Set and Coloring Problems with Its Application to Storing Problem
}

\author{
Min Meng and Jun-e Feng \\ School of Mathematics, Shandong University, Jinan 250100, China \\ Correspondence should be addressed to Jun-e Feng; fengjune@sdu.edu.cn \\ Received 10 January 2014; Accepted 21 March 2014; Published 17 April 2014 \\ Academic Editor: Yuri N. Sotskov
}

Copyright ( 2014 M. Meng and J.-e. Feng. This is an open access article distributed under the Creative Commons Attribution License, which permits unrestricted use, distribution, and reproduction in any medium, provided the original work is properly cited.

\begin{abstract}
This paper considers the stable set and coloring problems of hypergraphs and presents several new results and algorithms using the semitensor product of matrices. By the definitions of an incidence matrix of a hypergraph and characteristic logical vector of a vertex subset, an equivalent algebraic condition is established for hypergraph stable sets, as well as a new algorithm, which can be used to search all the stable sets of any hypergraph. Then, the vertex coloring problem is investigated, and a necessary and sufficient condition in the form of algebraic inequalities is derived. Furthermore, with an algorithm, all the coloring schemes and minimum coloring partitions with the given $q$ colors can be calculated for any hypergraph. Finally, one illustrative example and its application to storing problem are provided to show the effectiveness and applicability of the theoretical results.
\end{abstract}

\section{Introduction}

A hypergraph $H=(V, \mathscr{E})$ is composed of a finite set $V$ and a collection $\mathscr{E}$ of nonempty subsets of $V$, in which $V$ is called the vertex set of $H$ and $\mathscr{E}$ is called the edge set of $H$. Thus, graphs are a special kind of hypergraphs with two vertices in each edge. One of the basic problems about hypergraph theory is the stable set problem, which has been widely applied in many research fields like network coding $[1,2]$. Another basic problem about hypergraph theory is the coloring problem, which is one of NP-complete problems. There are various forms of hypergraph coloring such as vertex coloring, good coloring of edges, strong coloring, and equitable coloring. Graph coloring has been widely used in many real-life areas including scheduling and timetabling in engineering, register allocation in compilers, and air traffic flow management and frequency assignment in mobile [3-6]. The coloring problems of a special kind of graphs have been widely discussed in [7-9]. In recent years, there have been some references considering hypergraph theory, such as $[10,11]$. It has been successfully applied to many different areas such as Markov decision process [12], complete simple games [13], linear programming [14], and cooperation structures in games [15]. And a few references have analyzed the colorability of different kinds of hypergraphs [16-18]. However, there are no proper algebraic algorithms for stable set and coloring problems of hypergraphs. Thus, they are still open problems and it is necessary for us to establish new formulations and algorithms.

In recent years, Cheng et al. $[19,20]$ have proposed an effective tool, called the semitensor product (STP) of matrices. Via STP, Boolean networks can be converted into an algebraic form and many problems of Boolean networks, such as controllability and observability [21], fixed points and cycles [22], and control design problems [23-25], have been investigated. To learn more about the applications of STP, the readers can refer to [26-30].

In this paper, we investigate the stable set and vertex coloring problems of hypergraphs and present some new results and algorithms via STP. By incidence matrix and characteristic logical vector (CLV), a necessary and sufficient condition, as well as a new algorithm, is established for hypergraph stable sets. Then, we study the vertex coloring problem. An algebraic equivalent condition and an algorithm for coloring problem are obtained. With the two algorithms, we can calculate all the stable sets and coloring schemes with the given $q$ colors for any hypergraph. The results we obtained in this paper are feasible and clear, illustrated by an 
example and a practical application to the storing problems. Compared to [31], which has considered the stable set and coloring problems of graphs by STP, the results we obtained seem to be the generalization of [31]. However, just applying the results about graphs in [31] to hypergraphs, we cannot get the similar results about hypergraphs. In fact, there are many differences. We use incidence matrix of hypergraphs, while Wang et al. in [31] have used adjacent matrix of graphs. The derivations are completely different since the fundamental techniques used are not the same. Thus, in our paper, the results are new and innovative in some ways.

The remainder of this paper is organized as follows. Section 2 introduces the preliminaries on STP and hypergraph theory. In Sections 3 and 4, we investigate the stable set and coloring problems, respectively, and provide the main results and algorithms of this paper. One illustrative example is also given in Section 3, and the application of coloring problem to storing problem is presented in Section 5 to show the effectiveness and applicability of the obtained results. Section 6 makes a brief conclusion.

Before ending this section, we introduce some notations which will be used throughout this paper. Consider the following.

(i) $\mathscr{M}_{m \times n}$ is the set of $m \times n$ real matrices.

(ii) $\delta_{n}^{i}$ is the $i$ th column of the identity matrix $I_{n}$.

(iii) $\Delta_{n}:=\left\{\delta_{n}^{i} \mid i=1, \ldots, n\right\} . \Delta_{2}:=\Delta$.

(iv) $\mathscr{D}:=\{0,1\}$. Identify $1 \sim \delta_{2}^{1}, 0 \sim \delta_{2}^{2}$; then, $\mathscr{D} \sim \Delta$.

(v) $A \in \mathscr{M}_{m \times n}$ is called a Boolean matrix, if all its entries are either 0 or 1 . The set of $m \times n$ Boolean matrices is denoted by $\mathscr{B}_{m \times n}$.

(vi) A matrix $L \in \mathscr{M}_{n \times r}$ is called a logical matrix if the columns of $L$, denoted by $\operatorname{Col}(L)$, belong to $\Delta_{n}$. That is, $\operatorname{Col}(L) \subseteq \Delta_{n}$. And $\operatorname{Col}_{i}(L)$ means the ith column of $L$. Denote the set of $n \times r$ logical matrices by $\mathscr{L}_{n \times r}$.

(vii) If $L \in \mathscr{L}_{n \times r}$, by definition it can be expressed as $L=\left[\begin{array}{llll}\delta_{n}^{i_{1}} & \delta_{n}^{i_{2}} & \cdots & \delta_{n}^{i_{r}}\end{array}\right]$. Briefly, we denote it by $L=$ $\delta_{n}\left[\begin{array}{llll}i_{1} & i_{2} & \cdots & i_{r}\end{array}\right]$.

(viii) For $A=\left(a_{i j}\right), B=\left(b_{i j}\right) \in \mathscr{M}_{m \times n}, A \geq \geq(\leq \leq, \gg, \ll) B$ means $a_{i j} \geq(\leq,>,<) b_{i j}$, for all $i, j$.

(ix) For a set $S,|S|$ is the cardinality of $S$.

(x) $A=\operatorname{Diag}\left\{A_{1}, A_{2}, \ldots, A_{r}\right\}$ is a block-diagonal matrix with $A_{i}$ in the $(i, i)$ th position $(1 \leq i \leq r)$.

(xi) Let $A=\left(a_{i j}\right) \in \mathscr{M}_{m \times n}, B \in \mathscr{M}_{p \times q}$. The Kronecker product of matrices $A$ and $B$ is defined as

$$
A \otimes B:=\left[\begin{array}{cccc}
a_{11} B & a_{12} B & \cdots & a_{1 n} B \\
a_{21} B & a_{22} B & \cdots & a_{2 n} B \\
\vdots & \vdots & \ddots & \vdots \\
a_{m 1} B & a_{m 2} B & \cdots & a_{m n} B
\end{array}\right] .
$$

\section{Preliminaries}

In this section, we will give some necessary preliminaries on STP and hypergraph theory, which will be used later.
Definition 1 (see [20]). Let $A \in \mathscr{M}_{m \times n}$ and $B \in \mathscr{M}_{p \times q}$. The STP of matrices $A$ and $B$, denoted by $A \ltimes B$, is defined as

$$
A \ltimes B=\left(A \otimes I_{s / n}\right)\left(B \otimes I_{s / p}\right),
$$

where $s=\operatorname{lcm}\{n, p\}$ is the least common multiple of $n$ and $p$.

Remark 2. When $n=p$, STP coincides with conventional matrix product. So STP is a general form of matrix product. Throughout this paper, the matrix product is assumed to be STP, and the symbol " $\ltimes$ " is omitted if there is no confusion.

Definition 3 (see [19]). A swap matrix $W_{[m, n]}$ is an $m n \times m n$ matrix, defined as follows: label its columns by $(11,12, \ldots, 1 n, \ldots, m 1, m 2, \ldots, m n)$; label its rows by $(11,21, \ldots, m 1, \ldots, 1 n, 2 n, \ldots, m n)$, and then the element at the position $[(I, J),(i, j)]$ is

$$
w_{(I, J),(i, j)}=\delta_{i, j}^{I, J}= \begin{cases}1, & I=i, J=j, \\ 0, & \text { otherwise. }\end{cases}
$$

Definition 4 (see [32]). Let $A=\left(a_{i j}\right), B=\left(b_{i j}\right) \in M_{m \times n}$. The Hadamard product of $A$ and $B$ is defined as

$$
A \odot B=\left(a_{i j} b_{i j}\right) \in \mathscr{M}_{m \times n} .
$$

Lemma 5 (see [19]). (1) Let $X \in \mathscr{R}^{m}$ and $Y \in \mathscr{R}^{n}$ be two column vectors. Then,

$$
W_{[m, n]} X Y=Y X .
$$

(2) Given $A \in \mathscr{M}_{m \times n}$, let $Z \in \mathscr{R}^{t}$ be a column vector. Then,

$$
Z A=W_{[m, t]} A W_{[t, n]} Z=\left(I_{t} \otimes A\right) Z .
$$

(3) Let $X \in \mathscr{R}^{n}, Y \in \mathscr{R}^{q}$ be two column vectors and let $A \in \mathscr{M}_{m \times n}, B \in \mathscr{M}_{p \times q}$ be two given matrices. Then,

$$
(A X) \ltimes(B Y)=(A \otimes B)(X \ltimes Y) .
$$

(4) Let $f\left(x_{1}, x_{2}, \ldots, x_{r}\right)$ be a Boolean function. Then, there exists a unique logical matrix $L_{f} \in \mathscr{L}_{2 \times 2^{r}}$ such that

$$
f\left(x_{1}, x_{2}, \ldots, x_{r}\right)=L_{f} \ltimes_{i=1}^{r} x_{i} .
$$

Here, $L_{f} \in \mathscr{L}_{2 \times 2^{r}}$ is called the structure matrix of $f$.

Now, some structure matrices of basic logical operators are given as follows:

$$
\begin{aligned}
& M_{\vee}:=M_{d}=\delta_{2}\left[\begin{array}{llll}
1 & 1 & 1 & 2
\end{array}\right] ; \\
& M_{\wedge}:=M_{c}=\delta_{2}\left[\begin{array}{llll}
1 & 2 & 2 & 2
\end{array}\right] ; \\
& M_{\neg}:=M_{n}=\delta_{2}\left[\begin{array}{ll}
2 & 1
\end{array}\right] ; \\
& M_{\rightarrow}:=M_{i}=\delta_{2}\left[\begin{array}{llll}
1 & 2 & 1 & 1
\end{array}\right] .
\end{aligned}
$$

And the power reducing matrix is defined as

$$
M_{r}=\delta_{4}\left[\begin{array}{ll}
1 & 4
\end{array}\right] \text {. }
$$

Then, if $X, Y \in \Delta$, we will have $X \vee Y=M_{d} X Y, X \wedge Y=$ $M_{c} X Y, \neg X=M_{n} X, X \rightarrow Y=M_{i} X Y$, and $X \ltimes X=M_{r} X$. 
Definition 6 (see [33]). Let $V=\left\{v_{1}, v_{2}, \ldots, v_{n}\right\}$ be a finite set, and let $\mathscr{E}=\left\{E_{1}, E_{2}, \ldots, E_{m}\right\}$ be a family of subsets of $V$; that is, $E_{j} \subseteq V, j=1,2, \ldots, m$. The family $\mathscr{E}$ is said to be a hypergraph on $V$ denoted by $H=(V, \mathscr{E})$, if $E_{j} \neq \varnothing, j=$ $1,2, \ldots, m$, and $\bigcup_{j=1}^{m} E_{j}=V$. The elements $v_{1}, v_{2}, \ldots, v_{n}$ are called the vertices (hypervertices) and the sets $E_{1}, E_{2}, \ldots, E_{m}$ are called the edges (hyperedges).

The incidence matrix of hypergraph $H=(V, \mathscr{E})$ is a matrix $A=\left(a_{i j}\right)$ with $m$ rows that represent the edges of $H$ and $n$ columns that represent the vertices of $H$, such that

$$
a_{i j}= \begin{cases}1, & v_{j} \in E_{i}, \\ 0, & v_{j} \notin E_{i} .\end{cases}
$$

Definition 7 (see [33]). Given a hypergraph $H=(V, \mathscr{E})$, a set $S \subseteq V$ is called a stable set if it contains no edge $E_{i}$ with $\left|E_{i}\right|>$ 1. Furthermore, $S$ is called a maximum stable set, if any vertex subset strictly containing $S$ is not a stable set. A stable set $S$ is called an absolutely maximum stable set if $|S|$ is the largest among all of the stable sets of $H$. The stable number of $H$, denoted by $\alpha(H)$, is defined to be the maximum cardinality of all the stable sets of $H$.

Remark 8. For a hypergraph $H=(V, \mathscr{E})$, any subset of stable set $S$ is a stable set. If there exists $E_{i} \in \mathscr{E}$ satisfying $\left|E_{i}\right|=1$, that is, $H$ has an edge formed by an isolated vertex, then, all the stable sets of $H$ can be obtained from all the stable sets of $H^{\prime}=\left(V^{\prime}, \mathscr{E}^{\prime}\right)$ where $\mathscr{E}^{\prime}=\mathscr{E}-\left\{E_{i}\right\}$. In fact, if all the stable sets of $H^{\prime}$ are $S_{1}, \ldots, S_{r}$, then, all the stable sets of $H$ are $S_{1}, \ldots, S_{r}, S_{1} \cup\left\{E_{i}\right\}, \ldots, S_{r} \cup\left\{E_{i}\right\}$. Therefore, in this paper, we just consider the edges of cardinality more than one. Additionally, the empty set $\varnothing$ is regarded as a stable set of any hypergraph.

Definition 9 (see [33]). A q-coloring is defined to be a partition of $V$ into $q$ stable sets $S_{1}, S_{2}, \ldots, S_{q}$, each corresponding to a color. A hypergraph for which there exists a $q$-coloring is said to be $q$-colorable.

\section{Stable Set Problem}

In the section, we investigate the stable set problem of hypergraphs using the STP method and present algebraic equivalent conditions, as well as an algorithm.

Given a hypergraph $H=(V, \mathscr{E})$ with $n$ vertices $V=$ $\left\{v_{1}, v_{2}, \ldots, v_{n}\right\}$ and $m$ edges $\mathscr{E}=\left\{E_{1}, E_{2}, \ldots, E_{m}\right\}$, assume that the incidence matrix of $H$ is $A=\left(a_{i j}\right)_{m \times n}$. Denote the $i$ th row of $A$ by $a_{i}, i=1,2, \ldots, m$; then $A=\left[a_{1}^{T}, a_{2}^{T}, \ldots, a_{m}^{T}\right]^{T}$. Assume that $S$ is a subset of $V$. Then, in the following, we will discuss under what conditions the subset $S$ is a stable set. First, we define some vectors.

The CLV of $S$, denoted by $V_{S}=\left[x_{1}, x_{2}, \ldots, x_{n}\right]$, is denoted as

$$
x_{i}= \begin{cases}1, & v_{i} \in S, \\ 0, & v_{i} \notin S .\end{cases}
$$

And then denote

$$
\begin{array}{r}
y_{i j}=\left[\begin{array}{c}
a_{i j} \\
1-a_{i j}
\end{array}\right], \quad y_{j}=\left[\begin{array}{c}
x_{j} \\
1-x_{j}
\end{array}\right], \\
i=1,2, \ldots, m, \quad j=1,2, \ldots, n .
\end{array}
$$

It is easy to see that $V_{S}$ is a Boolean vector and $y_{i j}, y_{j} \in \Delta$. Then, we can present the following results.

Theorem 10. Consider the hypergraph $H=(V, \mathscr{E})$ expressed as above. Then $S$ is a stable set of $H$ if and only if the last row of matrix $\bar{M}$ has at least one zero element, where

$$
\begin{gathered}
\bar{M}=\left(\bigotimes_{j=1}^{n} M\right)\left(\ltimes_{k=1}^{n-1}\left(I_{2^{k}} \otimes W_{\left[2,2^{k}\right]}\right)\right)\left(\sum_{l=1}^{m} Y_{l}\right), \\
M=M_{n} M_{i}\left(I_{2} \otimes M_{c}\right) M_{r}, \quad Y_{l}=\ltimes_{t=1}^{n} y_{l t} .
\end{gathered}
$$

Proof. Let $\bar{E}_{l}=E_{l} \backslash S$ with the CLV $\bar{a}_{l}=\left[\bar{a}_{l 1}, \bar{a}_{l 2}, \ldots, \bar{a}_{l n}\right]$, $l=1,2, \ldots, m$. Denote

$$
\bar{y}_{l t}=\left[\begin{array}{c}
\bar{a}_{l t} \\
1-\bar{a}_{l t}
\end{array}\right], \quad l=1,2, \ldots, m, t=1,2, \ldots, n .
$$

Then, $S$ is a stable set if and only if, for every $l \in$ $\{1,2, \ldots, m\}, \bar{E}_{l} \neq \varnothing$; that is, $\bar{a}_{l} \neq 0_{1 \times n}$. Since $\bar{a}_{l} \neq 0_{1 \times n}$ if and only if $\ltimes_{t=1}^{n} \bar{y}_{l t} \neq \delta_{2^{n}}^{2^{n}}$ if and only if the last element of $\ltimes_{t=1}^{n} \bar{y}_{l t}$ is 0 , we just need to prove that, for every $l \in\{1,2, \ldots, m\}$, the last element of $\ltimes_{t=1}^{n} \bar{y}_{l t}$ is 0 if and only if the last row of matrix $\bar{M}$ has one zero component at least.

Let $J_{1}^{T}=\delta_{2^{n}}^{2^{n}}$. If, for every $l \in\{1,2, \ldots, m\}$, the last element of $\ltimes_{t=1}^{n} \bar{y}_{l t}$ is 0 , then, we get, for every $l, J_{1} \ltimes_{t=1}^{n} \bar{y}_{l t}=0$. Thus, $\bar{y}_{l t}$ satisfies

$$
J_{1} \sum_{l=1}^{m} \ltimes_{t=1}^{n} \bar{y}_{l t}=0
$$

Since $\bar{E}_{l}=E_{l} \backslash S, \bar{a}_{l t}=a_{l t}-a_{l t} \wedge x_{t}$. Hence,

$$
\begin{aligned}
\bar{y}_{l t} & =y_{l t}-y_{l t} \wedge y_{t}=\neg\left(y_{l t} \longrightarrow\left(y_{l t} \wedge y_{t}\right)\right) \\
& =M_{n} M_{i} y_{l t} M_{c} y_{l t} y_{t}=M_{n} M_{i}\left(I_{2} \otimes M_{c}\right) M_{r} y_{l t} y_{t} \\
& \triangleq M y_{l t} y_{t} .
\end{aligned}
$$

So (16) can be expressed as

$$
J_{1} \sum_{l=1}^{m} \ltimes_{t=1}^{n} M y_{l t} y_{t}=0 .
$$

By Lemma 5, we have

$$
\begin{aligned}
& \ltimes_{t=1}^{n} M y_{l t} y_{t}=\left(\bigotimes_{j=1}^{n} M\right)\left(\ltimes_{t=1}^{n} y_{l t} y_{t}\right), \\
& \ltimes_{t=1}^{n} y_{l t} y_{t}=y_{l 1} y_{1} y_{l 2} y_{2} \cdots y_{l n} y_{n} \\
&=y_{l_{1}} W_{[2,2]} y_{l 2} y_{1} y_{2} \cdots y_{l n} y_{n} \\
&=\left(I_{2} \otimes W_{[2,2]}\right) y_{l 1} y_{l 2} y_{1} y_{2} \cdots y_{l n} y_{n}=\cdots \\
&=\ltimes_{k=1}^{n-1}\left(I_{2^{k}} \otimes W_{\left[2,2^{k}\right]}\right) Y_{l} Y,
\end{aligned}
$$


where $Y=\ltimes_{t=1}^{n} y_{t} \in \Delta_{2^{n}}$ and $Y_{l}$ is described in (14). Therefore, (16) becomes

$$
J_{1} \sum_{l=1}^{m}\left(\bigotimes_{j=1}^{n} M\right) \ltimes_{k=1}^{n-1}\left(I_{2^{k}} \otimes W_{\left[2,2^{k}\right]}\right) Y_{l} Y=0
$$

That is,

$$
J_{1} \bar{M} Y=0
$$

Noticing that $Y \in \Delta_{2^{n}}$, we have that (21) having a solution $Y$ is equivalent to the last row of $\bar{M}$ having one zero element at least. The necessity is proved.

On the other hand, if the last row of $\bar{M}$ has one zero element at least, then the equation $J_{1} \bar{M} Y=0$ has a solution $Y \in \Delta_{2^{n}}$. Equivalently, (16) holds. Noticing that $J_{1} \ltimes_{t=1}^{n} \bar{y}_{l t} \geq 0$, from (16) we obtain $J_{1} \ltimes_{t=1}^{n} \bar{y}_{l t}=0$; that is, for every $l \in$ $\{1,2, \ldots, m\}$, the last element of $\ltimes_{t=1}^{n} \bar{y}_{l t}$ is 0 . Hence the proof is completed.

From the above theorem, we find that (21) plays an important role to calculate all the stable sets of hypergraph $H$. The following corollary shows the relationship between (21) and the stable sets of hypergraphs.

Corollary 11. Consider the hypergraph $H$ in Theorem 10. $H$ has a stable set if and only if (21) has a solution $Y=\ltimes_{t=1}^{n} y_{t} \in$ $\Delta_{2^{n}}$. In addition, the number of stable sets of $H$ is the cardinal of solution set of (21).

In order to obtain all the stable sets of any hypergraph, we will give an algorithm according to the proof of Theorem 10 and the results of Corollary 11.

Algorithm 12. Consider a hypergraph $H$ shown in Theorem 10. The following steps are given to find all the stable sets of $H$.

(1) Calculate the matrix $\bar{M}$ given in (14).

(2) Denote the last row of $\bar{M}$ by $\beta=\left[b_{1}, b_{2}, \ldots, b_{2^{n}}\right]$. If $b_{i} \neq 0$, for every $i \in\left\{1,2, \ldots, 2^{n}\right\}$, then, $H$ has no stable set and stop. Otherwise, find out all the zero elements of $\beta: b_{i_{1}}=b_{i_{2}}=\cdots=b_{i_{p}}=0$. Then, $b_{i_{k}}=0$ corresponds to a solution, $Y=\delta_{2^{n}}^{i_{k}}$, of (21) and so does a stable set of $H$.

(3) From $\ltimes_{j=1}^{n} y_{j}=\delta_{2^{n}}^{i_{k}}$, we can retrieve $y_{t}$ as $y_{t}=$ $S_{t}^{n} \ltimes_{j=1}^{n} y_{j}=S_{t}^{n} \delta_{2^{n}}^{i_{k}}, t=1,2, \ldots, n$, where $S_{t}^{n}, t=$ $1,2, \ldots, n$ are defined as follows [19]:

$$
\begin{gathered}
S_{1}^{n}=\delta_{2}[\underbrace{1 \cdots 1}_{2^{n-1}} \underbrace{2 \cdots 1}_{2^{n-1}}], \\
S_{2}^{n}=\delta_{2}[\underbrace{1 \cdots 1}_{2^{n-2}} \underbrace{2 \cdots 2}_{2^{n-2}} \underbrace{1 \cdots \cdots}_{2^{n-2}} \underbrace{2 \cdots \cdots}_{2^{n-2}}],
\end{gathered}
$$

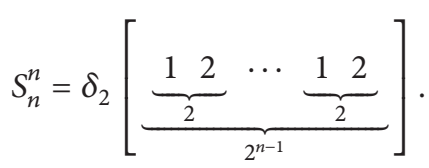

By the definition of (12), we obtain the stable set corresponding to $\delta_{2^{n}}^{i_{k}}$ :

$$
S\left(i_{k}\right)=\left\{v_{t} \mid y_{t}=\delta_{2}^{1}, 1 \leq t \leq n\right\} .
$$

Thus, all the stable sets of $H$ are $\left\{S\left(i_{k}\right) \mid k=\right.$ $1,2, \ldots, p\}$.

Remark 13. From Algorithm 12, we can obtain all the stable sets of hypergraph $H$; therefore, the absolutely maximum stable sets can also be determined. Denote the stable number of $H$ by $\alpha_{0}$; then, $\alpha_{0}=\max _{1 \leq k \leq p}\left\{\left|S\left(i_{k}\right)\right|\right\}$, and all the absolutely maximum stable sets can be derived as $S=\left\{S\left(i_{k}\right)|| S\left(i_{k}\right) \mid=\right.$ $\left.\alpha_{0}\right\}$.

Remark 14. Although the calculation of the above algorithm is complex, the advantage of this approach is that we can obtain all the stable sets as well as all the absolutely maximum stable sets of a hypergraph. And we can turn to the computer using MATLAB toolbox.

Example 15. Consider the hypergraph $H=(V, \mathscr{E})$, where $V=\left\{v_{1}, v_{2}, v_{3}, v_{4}, v_{5}\right\}, \mathscr{E}=\left\{E_{1}, E_{2}, E_{3}, E_{4}\right\}, E_{1}=\left\{v_{1}, v_{2}, v_{3}\right\}$, $E_{2}=\left\{v_{3}, v_{4}, v_{5}\right\}, E_{3}=\left\{v_{2}, v_{3}, v_{4}\right\}$, and $E_{4}=\left\{v_{2}, v_{4}, v_{5}\right\}$. In the following, we use Algorithm 12 to find all the stable sets of the hypergraph.

By the definition of the incidence matrix of the hypergraph $H$, the incidence matrix of $H$ is

$$
A=\left[\begin{array}{lllll}
1 & 1 & 1 & 0 & 0 \\
0 & 0 & 1 & 1 & 1 \\
0 & 1 & 1 & 1 & 0 \\
0 & 1 & 0 & 1 & 1
\end{array}\right]
$$

By MATLAB toolbox, we easily get

$$
\begin{gathered}
M=\left[\begin{array}{llll}
0 & 1 & 0 & 0 \\
1 & 0 & 1 & 1
\end{array}\right], \\
Y_{1}=\delta_{2^{5}}^{4}, \quad Y_{2}=\delta_{2^{5}}^{25}, \\
Y_{3}=\delta_{2^{5}}^{18}, \quad Y_{4}=\delta_{2^{5}}^{21} .
\end{gathered}
$$

Thus,

$$
\begin{aligned}
\sum_{l=1}^{4} Y_{l} & =\delta_{2^{5}}^{4}+\delta_{2^{5}}^{25}+\delta_{2^{5}}^{18}+\delta_{2^{5}}^{21} \\
& =\left[\begin{array}{lllllllllllllllll}
0 & 0 & 0 & 1 & 0 & 0 & 0 & 0 & 0 & 0 & 0 & 0 & 0 & 0 & 0 & 0 & 0 \\
1 & 0 & 0 & 1 & 0 & 0 & 0 & 1 & 0 & 0 & 0 & 0 & 0 & 0 & 0
\end{array}\right]^{T} .
\end{aligned}
$$


Then, we obtain the last row of $\bar{M}$ as

$$
\beta=\left[\begin{array}{llllllllllllllll}
4 & 2 & 1 & 1 & 1 & 0 & 0 & 0 & 1 & 0 & 0 & 0 & 0 & 0 & 0 & 0
\end{array}\right.
$$

$\left.\begin{array}{llllllllllllllll}3 & 1 & 0 & 0 & 1 & 0 & 0 & 0 & 1 & 0 & 0 & 0 & 0 & 0 & 0 & 0\end{array}\right]$,

and the indexes of zero elements in $\beta$ are

$$
\begin{aligned}
P & =\left\{i_{k} \mid \beta\left(i_{k}\right)=0\right\} \\
& =\{6,7,8,10,11,12,13,14,15,16,19,20,22,
\end{aligned}
$$

$23,24,26,27,28,29,30,31,32\}$.

For each index $i_{k} \in P$, let $\ltimes_{i=1}^{5} y_{i}=\delta_{2^{5}}^{i_{k}}$. Via computing $y_{i}=$ $S_{i}^{5} \delta_{2^{5}}^{i_{k}}, i=1,2, \ldots, 5$, we have all the stable sets of $H$ as follows:

$$
\begin{aligned}
i_{1} & =6 \sim\left(x_{1}, x_{2}, x_{3}, x_{4}, x_{5}\right) \\
& =(1,1,0,1,0) \sim S_{1}=\left\{v_{1}, v_{2}, v_{4}\right\}, \\
i_{2} & =7 \sim\left(x_{1}, x_{2}, x_{3}, x_{4}, x_{5}\right) \\
& =(1,1,0,0,1) \sim S_{2}=\left\{v_{1}, v_{2}, v_{5}\right\}, \\
i_{3} & =8 \sim\left(x_{1}, x_{2}, x_{3}, x_{4}, x_{5}\right) \\
& =(1,1,0,0,0) \sim S_{3}=\left\{v_{1}, v_{2}\right\}, \\
i_{4} & =10 \sim\left(x_{1}, x_{2}, x_{3}, x_{4}, x_{5}\right) \\
& =(1,0,1,1,0) \sim S_{4}=\left\{v_{1}, v_{3}, v_{4}\right\}, \\
i_{5} & =11 \sim\left(x_{1}, x_{2}, x_{3}, x_{4}, x_{5}\right) \\
& =(1,0,1,0,1) \sim S_{5}=\left\{v_{1}, v_{3}, v_{5}\right\}, \\
i_{6} & =12 \sim\left(x_{1}, x_{2}, x_{3}, x_{4}, x_{5}\right) \\
& =(1,0,1,0,0) \sim S_{6}=\left\{v_{1}, v_{3}\right\}, \\
i_{7} & =13 \sim\left(x_{1}, x_{2}, x_{3}, x_{4}, x_{5}\right) \\
& =(1,0,0,1,1) \sim S_{7}=\left\{v_{1}, v_{4}, v_{5}\right\}, \\
i_{8} & =14 \sim\left(x_{1}, x_{2}, x_{3}, x_{4}, x_{5}\right) \\
& =(1,0,0,1,0) \sim S_{8}=\left\{v_{1}, v_{4}\right\}, \\
i_{9} & =15 \sim\left(x_{1}, x_{2}, x_{3}, x_{4}, x_{5}\right) \\
& =(1,0,0,0,1) \sim S_{9}=\left\{v_{1}, v_{5}\right\}, \\
i_{10} & =16 \sim\left(x_{1}, x_{2}, x_{3}, x_{4}, x_{5}\right) \\
& =(1,0,0,0,0) \sim S_{10}=\left\{v_{1}\right\}, \\
i_{11} & =19 \sim\left(x_{1}, x_{2}, x_{3}, x_{4}, x_{5}\right) \\
& =(0,1,1,0,1) \sim S_{11}=\left\{v_{2}, v_{3}, v_{5}\right\},
\end{aligned}
$$

$$
\begin{aligned}
i_{12} & =20 \sim\left(x_{1}, x_{2}, x_{3}, x_{4}, x_{5}\right) \\
& =(0,1,1,0,0) \sim S_{12}=\left\{v_{2}, v_{3}\right\}, \\
i_{13} & =22 \sim\left(x_{1}, x_{2}, x_{3}, x_{4}, x_{5}\right) \\
& =(0,1,0,1,0) \sim S_{13}=\left\{v_{2}, v_{4}\right\}, \\
i_{14} & =23 \sim\left(x_{1}, x_{2}, x_{3}, x_{4}, x_{5}\right) \\
& =(0,1,0,0,1) \sim S_{14}=\left\{v_{2}, v_{5}\right\}, \\
i_{15} & =24 \sim\left(x_{1}, x_{2}, x_{3}, x_{4}, x_{5}\right) \\
& =(0,1,0,0,0) \sim S_{15}=\left\{v_{2}\right\}, \\
i_{16} & =26 \sim\left(x_{1}, x_{2}, x_{3}, x_{4}, x_{5}\right) \\
& =(0,0,1,1,0) \sim S_{16}=\left\{v_{3}, v_{4}\right\}, \\
i_{17} & =27 \sim\left(x_{1}, x_{2}, x_{3}, x_{4}, x_{5}\right) \\
& =(0,0,1,0,1) \sim S_{17}=\left\{v_{3}, v_{5}\right\}, \\
i_{18} & =28 \sim\left(x_{1}, x_{2}, x_{3}, x_{4}, x_{5}\right) \\
& =(0,0,1,0,0) \sim S_{18}=\left\{v_{3}\right\}, \\
i_{19} & =29 \sim\left(x_{1}, x_{2}, x_{3}, x_{4}, x_{5}\right) \\
& =(0,0,0,1,1) \sim S_{19}=\left\{v_{4}, v_{5}\right\}, \\
i_{20} & =30 \sim\left(x_{1}, x_{2}, x_{3}, x_{4}, x_{5}\right) \\
& =(0,0,0,1,0) \sim S_{20}=\left\{v_{4}\right\}, \\
i_{21} & =31 \sim\left(x_{1}, x_{2}, x_{3}, x_{4}, x_{5}\right) \\
& =(0,0,0,0,1) \sim S_{21}=\left\{v_{5}\right\}, \\
i_{22} & =32 \sim\left(x_{1}, x_{2}, x_{3}, x_{4}, x_{5}\right) \\
& =(0,0,0,0,0) \sim S_{22}=\varnothing .
\end{aligned}
$$

Therefore, from the calculation results, we know $\max _{i_{k}}\left\{\left|S_{i_{k}}\right|\right\}=3$; all the absolutely maximum stable sets are $\left\{S_{1}, S_{2}, S_{4}, S_{5}, S_{7}, S_{11}\right\}$.

\section{Coloring Problem}

In this section, we study the vertex coloring problem of hypergraphs, that is, given $q$ colors, how to color the vertices of the hypergraph $H$ such that no edge has all its vertices with the same color.

Consider a hypergraph $H=(V, \mathscr{E})$, with $V=$ $\left\{v_{1}, v_{2}, \ldots, v_{n}\right\}, \mathscr{E}=\left\{E_{1}, E_{2}, \ldots, E_{m}\right\}$, and assume that its incidence matrix is $A=\left(a_{i j}\right) \in \mathscr{B}_{m \times n}$. Given $q$ kinds of colors: $c_{1}, c_{2}, \ldots, c_{q}$, let $N=\left\{c_{1}, c_{2}, \ldots, c_{q}\right\}$ be the color set; then, we can define a mapping $\phi: V \mapsto N$. Since all of the $q$ colors may not be used, $\phi$ may not be a surjective. To solve the coloring problem of hypergraph $H$, we can just find 
a mapping $\phi$ satisfying that, for every edge $E_{k}$, there are two different vertices $v_{s}, v_{t} \in E_{k}$ satisfying $\phi\left(v_{s}\right) \neq \phi\left(v_{t}\right)$.

If the color problem is solvable, then each vertex in $V$ has been colored by one of the colors. Thus, for given $q$ colors, we can define a CLV $x_{t}=\delta_{q}^{s} \in \Delta_{q}$ corresponding to the vertex $v_{t} \in V$ with the color $c_{s}$; that is, $\phi\left(v_{t}\right)=c_{s}$. Before investigating the coloring problem of $H$, we first calculate the structure matrix of the $q$ valued function $x_{s} \odot x_{t}$ for $x_{s}, x_{t} \in \Delta_{q}$.

Similar to (12), the $q$ valued retrievers can be given as [19]

$$
\begin{aligned}
& S_{1, q}^{n}=\delta_{q}[\underbrace{1 \cdots}_{q^{n-1}} \underbrace{2 \cdots}_{q^{n-1}} \cdots \underbrace{q \cdots}_{q^{n-1}}], \\
& S_{2, q}^{n}=\delta_{q}[\underbrace{\underbrace{1 \cdots 1}_{q^{n-2}} \underbrace{2 \cdots}_{q^{n-2}} \cdots \underbrace{q \cdots}_{q^{n-2}} \underbrace{q^{2}}_{q^{n-2}} \cdots \underbrace{1 \cdots 2}_{q^{n-2}} \cdots \underbrace{1 \cdots}_{q^{n-2}}}_{q}] \text {, }
\end{aligned}
$$

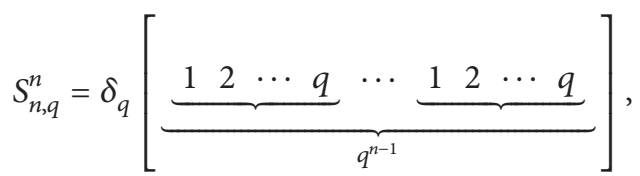

and then, $x_{s}=S_{s, q}^{n} \ltimes_{i=1}^{n} x_{i}$ and $x_{t}=S_{t, q}^{n} \ltimes_{i=1}^{n} x_{i}$. Let $M_{r, q^{n}}=$ $\operatorname{Diag}\left\{\delta_{q^{n}}^{1}, \delta_{q^{n}}^{2}, \ldots, \delta_{q^{n}}^{q^{n}}\right\}$ and $H_{q}=\operatorname{Diag}\left\{e_{1}, e_{2}, \ldots, e_{q}\right\}$, where $e_{i}=\left(\delta_{q}^{i}\right)^{T}$. With simple calculation, we have

$$
\begin{aligned}
x_{s} \odot x_{t} & =H_{q} x_{s} \ltimes x_{t}=H_{q} S_{s, q}^{n}\left(\ltimes_{i=1}^{n} x_{i}\right) S_{t, q}^{n}\left(\ltimes_{i=1}^{n} x_{i}\right) \\
& =H_{q} S_{s, q}^{n}\left(I_{q^{n}} \otimes S_{t, q}^{n}\right) M_{r, q^{n}}\left(\ltimes_{i=1}^{n} x_{i}\right) .
\end{aligned}
$$

Define the structure matrix of $\odot$ by

$$
M_{s t}^{H}=H_{q} S_{s, q}^{n}\left(I_{q^{n}} \otimes S_{t, q}^{n}\right) M_{r, q^{n}}
$$

Next, we give the following algebraic condition of coloring problem of hypergraph $H$.

Theorem 16. The coloring problem is solvable if and only if there exists $j \in\left\{1,2, \ldots, q^{n}\right\}$ such that

$$
\mathrm{Col}_{j}(M) \ll b,
$$

where

$$
\begin{aligned}
& b=\sum_{s=1}^{n-1} \sum_{t=s+1}^{n}\left[\begin{array}{c}
a_{1 s} a_{1 t} \\
a_{2 s} a_{2 t} \\
\vdots \\
a_{m s} a_{m t}
\end{array}\right], \\
& M=\sum_{s=1}^{n-1} \sum_{t=s+1}^{n}\left[\begin{array}{c}
a_{1 s} a_{1 t} \\
a_{2 s} a_{2 t} \\
\vdots \\
a_{m s} a_{m t}
\end{array}\right] J_{2} M_{s t}^{H},
\end{aligned}
$$

$J_{2}=[1,1, \ldots, 1]_{q}$, and $M_{s t}^{H}$, a structure matrix of $\odot$, is $H_{q} S_{s, q}^{n}\left(I_{q^{n}} \otimes S_{t, q}^{n}\right) M_{r, q^{n}}$ by $(31)$.
Proof. (Necessity) If the coloring problem is solvable, then, for every edge $E_{k} \in \mathscr{E}$, there exist two different vertices $v_{s}, v_{t}$ in $E_{k}$ such that $\phi\left(v_{s}\right) \neq \phi\left(v_{t}\right)$. That is, $a_{k s}=a_{k t}=1$ but $x_{s} \neq x_{t}$. Then, for each $k \in\{1,2, \ldots, m\}$, there exist $s, t \in\{1,2, \ldots, n\}$, $s \neq t$, satisfying $a_{k s}=a_{k t}=1$ but $x_{s} \neq x_{t}$, which implies $J_{2} x_{s} \odot$ $x_{t}=0<1$. Thus,

$$
\sum_{s=1}^{n-1} \sum_{t=s+1}^{n} a_{k s} a_{k t} J_{2} x_{s} \odot x_{t}<\sum_{s=1}^{n-1} \sum_{t=s+1}^{n} a_{k s} a_{k t} .
$$

By (31), we have

$$
\begin{aligned}
& \sum_{s=1}^{n-1} \sum_{t=s+1}^{n} a_{k s} a_{k t} J_{2} M_{s t}^{H}\left(\ltimes_{i=1}^{n} x_{i}\right) \\
& \quad<\sum_{s=1}^{n-1} \sum_{t=s+1}^{n} a_{k s} a_{k t}, \quad \forall k \in\{1,2, \ldots, m\} .
\end{aligned}
$$

Equivalently,

$$
M Y \ll b,
$$

where $Y=\ltimes_{i=1}^{n} x_{i}$. Considering that $Y=\ltimes_{i=1}^{n} x_{i} \in \Delta_{q^{n}}$, from the inequality (37), we know that there exists $j \in\left\{1,2, \ldots, q^{n}\right\}$ such that $\operatorname{Col}_{j}(M) \ll b$.

(Sufficiency) Assume that there exists $j \in\left\{1,2, \ldots, q^{n}\right\}$ such that $\operatorname{Col}_{j}(M) \ll b$. Then the inequality (37) has a solution $Y \in \Delta_{q^{n}}$. Thus, for every $k \in\{1,2, \ldots, m\}$, the inequality (35) holds. Noticing that $0 \leq J_{2} x_{s} \odot x_{t} \leq 1$, we get that, for each $k \in\{1,2, \ldots, m\}$, there exist $s, t \in$ $\{1,2, \ldots, n\}, s \neq t$ such that $a_{k s}=a_{k t}=1$ implies $J_{2} x_{s} \odot x_{t}=$ $0<1$. Hence, the proof is completed.

Based on the algebraic equivalent condition of the coloring problem, we can construct an algorithm to find out all the coloring schemes and coloring partitions. 
Algorithm 17. Consider the hypergraph $H$ and let a color set $N=\left\{c_{1}, c_{2}, \ldots, c_{q}\right\}$. Each vertex $v_{t}$ corresponds to a color and, thus, corresponds to a $q$ valued CLV $x_{t} \in \Delta_{q}$. We give the following steps to calculate all the coloring schemes.

(1) Calculate the matrices $M$ and $b$ given in Theorem 16.

(2) Find if there exists $j \in\left\{1,2, \ldots, q^{n}\right\}$ such that $\mathrm{Col}_{j}(M) \ll b$. If not, the coloring problem with the $q$ colors is not solvable, and stop. Otherwise, find out all the columns of $M$ satisfying the inequality (33) and denote their indexes by the set

$$
Q=\left\{j \mid \operatorname{Col}_{j}(M) \ll b\right\} .
$$

(3) Let $\ltimes_{l=1}^{n} x_{l}=\delta_{q^{n}}^{j}$, for every $j \in Q$. By (30), we get $x_{i}=$ $S_{i, q}^{n}\left(\ltimes_{l=1}^{n} x_{l}\right)=S_{i, q}^{n} \delta_{q^{n}}^{j}, i=1,2, \ldots, n$. Then, let

$$
\begin{aligned}
& S_{c_{1}}^{j}=\left\{v_{i} \mid x_{i}=\delta_{q}^{1}, 1 \leq i \leq n\right\}, \\
& S_{\mathcal{C}_{2}}^{j}=\left\{v_{i} \mid x_{i}=\delta_{q}^{2}, 1 \leq i \leq n\right\},
\end{aligned}
$$

$$
S_{c_{q}}^{j}=\left\{v_{i} \mid x_{i}=\delta_{q}^{q}, 1 \leq i \leq n\right\},
$$

where $S_{c_{k}}^{j}$ is the set of vertices colored by the $k$ th color, $k \in\{1,2, \ldots, q\}$. Then, there are $|Q|$ kinds of coloring schemes corresponding to the elements in $Q$.

With the result of Theorem 16, we can easily obtain a corollary on $S_{\mathcal{c}_{k}}^{j}, k \in\{1,2, \ldots, q\}, j \in Q$.

Corollary 18. Assume that there exists $j \in\left\{1,2, \ldots, q^{n}\right\}$ such that $\mathrm{Col}_{j}(M) \ll b$. Then, the following hold:

(1) for each $k \in\{1,2, \ldots, q\}, S_{\mathcal{c}_{k}}^{j}$ defined in Algorithm 17 is a stable set of $H$,

(2) for each $j \in Q,\left\{S_{c_{1}}^{j}, S_{c_{2}}^{j}, \ldots, S_{c_{q}}^{j}\right\}$ is a partition of the vertices of $H$.

Remark 19. From Theorem 16 and Corollary 18, we know that if the coloring problem is solvable with the given $q$ colors, the hypergraph is q-colorable. On the other hand, given the $q$ colors, the coloring schemes obtained in Algorithm 17 contain all the coloring schemes with the colors not more than $q$. Then, the chromatic number denoted by $\chi(H)$, which is the minimum number of colors being used, is $\chi(H)=$ $\min _{j \in Q}\left\{\left|N_{j}\right|\right\}$, where $N_{j}=\left\{i \mid S_{c_{i}}^{j} \neq \varnothing, 1 \leq i \leq q\right\}$. Therefore, the minimum coloring partition is

$$
\left\{S_{c_{1}}^{j_{0}}, S_{c_{2}}^{j_{0}}, \ldots, S_{c_{q}}^{j_{0}}\right\} \backslash\left\{S_{c_{i}}^{j} \mid S_{c_{i}}^{j}=\varnothing\right\}, \quad \text { where } N_{j_{0}}=\chi(H)
$$

\section{Application in Storing Problem}

A company produces $n$ kinds of chemicals which contain some products that cannot be put in the same storehouse. The problem is that how many storehouses are needed at least to store the $n$ kinds of chemicals and how to assign them. In order to solve the problem, we denote the $n$ kinds of chemicals by $V=\left\{v_{1}, v_{2}, \ldots, v_{n}\right\}$ and $m$ kinds of circumstances by $\mathscr{E}=\left\{E_{1}, E_{2}, \ldots, E_{m}\right\}$ where the chemicals in $E_{i} \subseteq V$, $i=1,2, \ldots, m$, cannot be put in the same storehouse. Immediately, we obtain a hypergraph $H=(V, \mathscr{E})$. Then some chemicals can be put in the same storehouse if and only if the vertices corresponding to the chemicals can be colored with the same color. Therefore, to assign these chemicals is equivalent to solve the coloring problem of $H$. Here, we will give the following numerical example to illustrate it.

Example 20. There are five kinds of chemicals denoted by $V=\left\{v_{1}, v_{2}, v_{3}, v_{4}, v_{5}\right\}$ needed to be put into two storehouses. Let a hypergraph $H$ have the vertex set as $V$. And we know that some dangerous thing will happen if the following combinations appear: $\left\{v_{1}, v_{2}, v_{3}\right\},\left\{v_{3}, v_{4}, v_{5}\right\},\left\{v_{2}, v_{3}, v_{4}\right\}$, and $\left\{v_{2}, v_{4}, v_{5}\right\}$. Then we consider that these combinations are edges of the hypergraph. Thus, the storing problem is equivalent to the hypergraph coloring problem with two different colors. Letting a two-color set $N=\left\{c_{1}=\operatorname{Red}, c_{2}=\right.$ Blue $\}$, by Algorithm 17, we can get all the coloring schemes.

The incidence matrix of the hypergraph $H$ is as follows:

$$
A=\left[\begin{array}{lllll}
1 & 1 & 1 & 0 & 0 \\
0 & 0 & 1 & 1 & 1 \\
0 & 1 & 1 & 1 & 0 \\
0 & 1 & 0 & 1 & 1
\end{array}\right]
$$

By Theorem 16, using MATLAB toolbox, we easily obtain

$$
b=\left[\begin{array}{llll}
3 & 3 & 3 & 3
\end{array}\right]^{T}
$$

$$
M=\left[\begin{array}{llllllllllllllllllllllllllllllll}
3 & 3 & 3 & 3 & 1 & 1 & 1 & 1 & 1 & 1 & 1 & 1 & 1 & 1 & 1 & 1 & 1 & 1 & 1 & 1 & 1 & 1 & 1 & 1 & 1 & 1 & 1 & 1 & 3 & 3 & 3 & 3 \\
3 & 1 & 1 & 1 & 1 & 1 & 1 & 3 & 3 & 1 & 1 & 1 & 1 & 1 & 1 & 3 & 3 & 1 & 1 & 1 & 1 & 1 & 1 & 3 & 3 & 1 & 1 & 1 & 1 & 1 & 1 & 3 \\
3 & 3 & 1 & 1 & 1 & 1 & 1 & 1 & 1 & 1 & 1 & 1 & 1 & 1 & 3 & 3 & 3 & 3 & 1 & 1 & 1 & 1 & 1 & 1 & 1 & 1 & 1 & 1 & 1 & 1 & 3 & 3 \\
3 & 1 & 1 & 1 & 3 & 1 & 1 & 1 & 1 & 1 & 1 & 3 & 1 & 1 & 1 & 3 & 3 & 1 & 1 & 1 & 3 & 1 & 1 & 1 & 1 & 1 & 1 & 3 & 1 & 1 & 1 & 3
\end{array}\right] .
$$


Then, the index set $Q$ of $j$ satisfying (33) is

$$
\begin{aligned}
Q & =\left\{j \mid \operatorname{Col}_{j}(M) \ll b\right\} \\
& =\{6,7,10,11,13,14,19,20,22,23,26,27\} .
\end{aligned}
$$

For each $j \in Q$, let $\ltimes_{i=1}^{5} x_{i}=\delta_{2^{5}}^{j}$. By computing $x_{i}=$ $S_{i, 2}^{5} \delta_{2^{5}}^{j}, i=1,2, \ldots, 5$, we have

$$
j=6, \quad \delta_{2^{5}}^{6} \sim\left[x_{1}, x_{2}, x_{3}, x_{4}, x_{5}\right]=\delta_{2}\left[\begin{array}{lllll}
1 & 1 & 0 & 1 & 0
\end{array}\right],
$$$$
j=7, \quad \delta_{2^{5}}^{7} \sim\left[x_{1}, x_{2}, x_{3}, x_{4}, x_{5}\right]=\delta_{2}\left[\begin{array}{lllll}
1 & 1 & 0 & 0 & 1
\end{array}\right],
$$$$
j=10, \quad \delta_{2^{5}}^{10} \sim\left[x_{1}, x_{2}, x_{3}, x_{4}, x_{5}\right]=\delta_{2}\left[\begin{array}{lllll}
1 & 0 & 1 & 1 & 0
\end{array}\right],
$$$$
j=11, \quad \delta_{2^{5}}^{11} \sim\left[x_{1}, x_{2}, x_{3}, x_{4}, x_{5}\right]=\delta_{2}\left[\begin{array}{lllll}
1 & 0 & 1 & 0 & 1
\end{array}\right],
$$$$
j=13, \quad \delta_{2^{5}}^{13} \sim\left[x_{1}, x_{2}, x_{3}, x_{4}, x_{5}\right]=\delta_{2}\left[\begin{array}{lllll}
1 & 0 & 0 & 1 & 1
\end{array}\right],
$$$$
j=14, \quad \delta_{2^{5}}^{14} \sim\left[x_{1}, x_{2}, x_{3}, x_{4}, x_{5}\right]=\delta_{2}\left[\begin{array}{lllll}
1 & 0 & 0 & 1 & 0
\end{array}\right],
$$$$
j=19, \quad \delta_{2^{5}}^{19} \sim\left[x_{1}, x_{2}, x_{3}, x_{4}, x_{5}\right]=\delta_{2}\left[\begin{array}{lllll}
0 & 1 & 1 & 0 & 1
\end{array}\right],
$$$$
j=20, \quad \delta_{2^{5}}^{20} \sim\left[x_{1}, x_{2}, x_{3}, x_{4}, x_{5}\right]=\delta_{2}\left[\begin{array}{lllll}
0 & 1 & 1 & 0 & 0
\end{array}\right],
$$$$
j=22, \quad \delta_{2^{5}}^{22} \sim\left[x_{1}, x_{2}, x_{3}, x_{4}, x_{5}\right]=\delta_{2}\left[\begin{array}{lllll}
0 & 1 & 0 & 1 & 0
\end{array}\right],
$$$$
j=23, \quad \delta_{2^{5}}^{23} \sim\left[x_{1}, x_{2}, x_{3}, x_{4}, x_{5}\right]=\delta_{2}\left[\begin{array}{lllll}
0 & 1 & 0 & 0 & 1
\end{array}\right],
$$$$
j=26, \quad \delta_{2^{5}}^{26} \sim\left[x_{1}, x_{2}, x_{3}, x_{4}, x_{5}\right]=\delta_{2}\left[\begin{array}{lllll}
0 & 0 & 1 & 1 & 0
\end{array}\right],
$$$$
j=27, \quad \delta_{2^{5}}^{27} \sim\left[x_{1}, x_{2}, x_{3}, x_{4}, x_{5}\right]=\delta_{2}\left[\begin{array}{lllll}
0 & 0 & 1 & 0 & 1
\end{array}\right],
$$

from which we obtain the following 12 coloring schemes:

Scheme 1: $\quad S_{c_{1}}^{6}=\left\{v_{1}, v_{2}, v_{4}\right\}($ Red $)$,

$$
S_{c_{2}}^{8}=\left\{v_{3}, v_{5}\right\} \text { (Blue) ; }
$$

Scheme 2: $\quad S_{c_{1}}^{7}=\left\{v_{1}, v_{2}, v_{5}\right\}(\operatorname{Red})$,

$$
S_{c_{2}}^{8}=\left\{v_{3}, v_{4}\right\} \text { (Blue); }
$$

Scheme 3: $\quad S_{c_{1}}^{10}=\left\{v_{1}, v_{3}, v_{4}\right\}(\operatorname{Red})$,

$$
S_{c_{2}}^{8}=\left\{v_{2}, v_{5}\right\} \text { (Blue); }
$$

Scheme 4: $\quad S_{c_{1}}^{11}=\left\{v_{1}, v_{3}, v_{5}\right\}(\operatorname{Red})$,

$$
S_{c_{2}}^{8}=\left\{v_{2}, v_{4}\right\} \text { (Blue); }
$$

Scheme 5: $\quad S_{c_{1}}^{13}=\left\{v_{1}, v_{3}, v_{5}\right\}($ Red $)$,

$$
S_{c_{2}}^{8}=\left\{v_{2}, v_{4}\right\} \text { (Blue); }
$$

Scheme 6: $\quad S_{c_{1}}^{14}=\left\{v_{1}, v_{4}\right\}(\mathrm{Red})$,

$$
S_{c_{2}}^{8}=\left\{v_{2}, v_{3}, v_{5}\right\} \text { (Blue); }
$$

Scheme $7: \quad S_{c_{1}}^{19}=\left\{v_{2}, v_{3}, v_{5}\right\}$ (Red),

$$
S_{c_{2}}^{8}=\left\{v_{1}, v_{4}\right\} \text { (Blue); }
$$

Scheme 8: $\quad S_{c_{1}}^{20}=\left\{v_{2}, v_{3}\right\}($ Red $)$,

$$
S_{c_{2}}^{8}=\left\{v_{1}, v_{4}, v_{5}\right\} \text { (Blue); }
$$

Scheme 9: $\quad S_{c_{1}}^{22}=\left\{v_{2}, v_{4}\right\}($ Red $)$,

$$
S_{c_{2}}^{8}=\left\{v_{1}, v_{3}, v_{5}\right\} \text { (Blue); }
$$

Scheme 10: $\quad S_{c_{1}}^{23}=\left\{v_{2}, v_{5}\right\}($ Red $)$,

$$
S_{c_{2}}^{8}=\left\{v_{1}, v_{3}, v_{4}\right\} \text { (Blue); }
$$

Scheme 11: $\quad S_{c_{1}}^{26}=\left\{v_{3}, v_{4}\right\}($ Red $)$,

$$
S_{c_{2}}^{8}=\left\{v_{1}, v_{2}, v_{5}\right\} \text { (Blue); }
$$

Scheme 12: $\quad S_{c_{1}}^{27}=\left\{v_{3}, v_{5}\right\}$ (Red),

$$
S_{c_{2}}^{8}=\left\{v_{1}, v_{2}, v_{4}\right\} \text { (Blue). }
$$

Thus, there are totally 12 kinds of storing methods.

\section{Conclusion}

In this paper, the stable set and vertex coloring problems of hypergraphs have been revised. Several new results and algorithms have been presented via a method of STP. By defining the incidence matrix of hypergraph and CLV of a vertex subset, one equivalent condition has been established for hypergraph stable set. And a new algorithm to find out all the stable sets and all the absolutely maximum stable sets has been obtained. Furthermore, we have considered the vertex coloring problem and got a necessary and sufficient condition in the form of algebraic inequality, by which an algorithm has been derived to search all the coloring schemes and minimum coloring partitions with the given $q$ colors for any hypergraph. Finally, the illustrative example and the application to storing problem have shown that the results presented in this paper are very effective. In papers $[34,35]$, the scheduling jobs can induce a mixed graph coloring, not a hypergraph coloring. Thus, the mixed graph coloring problem will be interesting to be discussed by STP in the future.

\section{Conflict of Interests}

The authors declare that there is no conflict of interests regarding the publication of this paper.

\section{Acknowledgments}

The work was partially supported by NNSF of China (61374025), Research Awards Young and Middle-Aged Scientists of Shandong Province (BS2011SF009, BS2011DX019), 
Excellent Youth Foundation of Shandongs Natural Scientific Committee (JQ201219).

\section{References}

[1] D. Traskov, M. Heindlmaie, M. Médard, R. Koetter, and D. S. Lun, "Scheduling for network coded multicast: a conflict graph formulation," in Proceedings of the IEEE GLOBECOM Workshops, pp. 1-5, New Orleans, La, USA, December 2008.

[2] M. Yang and Y. Yang, "A hypergraph approach to linear network coding in multicast networks," IEEE Transactions on Parallel and Distributed Systems, vol. 21, no. 7, pp. 968-982, 2010.

[3] N. Barnier and P. Brisset, "Graph coloring for air traffic flow management," Annals of Operations Research, vol. 130, no. 1-4, pp. 163-178, 2004.

[4] M. W. Carter, G. Laporte, and S. Y. Lee, "Examination timetabling: algorithmic strategies and applications," Journal of the Operational Research Society, vol. 47, no. 3, pp. 373-383, 1996.

[5] G. J. Chaitin, M. A. Auslander, A. K. Chandra, J. Cocke, M. E. Hopkins, and P. W. Markstein, "Register allocation via coloring," Computer Languages, vol. 6, no. 1, pp. 47-57, 1981.

[6] A. Gamst, "Some lower bounds for a class of frequency assignment problems," IEEE Transactions on Vehicular Technology, vol. 35, no. 1, pp. 8-14, 1986.

[7] B. Wang and J.-L. Wu, "Total colorings of planar graphs without intersecting 5-cycles," Discrete Applied Mathematics, vol. 160, no. 12, pp. 1815-1821, 2012.

[8] B. Wang and J.-L. Wu, "Total colorings of planar graphs with maximum degree seven and without intersecting 3-cycles," Discrete Mathematics, vol. 311, no. 18-19, pp. 2025-2030, 2011.

[9] X. Zhang and J.-L. Wu, "On edge colorings of 1-planar graphs," Information Processing Letters, vol. 111, no. 3, pp. 124-128, 2011.

[10] H. Shen, S. Lv, X. Dong, J. Deng, X. Wang, and X. Zhou, "Hypergraph modeling and approximation algorithms for the minimum length link scheduling in multiuser MIMO networks," Journal of Applied Mathematics, vol. 2013, Article ID 982713, 9 pages, 2013.

[11] F. M. Malvestuto, "Decomposable convexities in graphs and hypergraphs," ISRN Combinatorics, vol. 2013, Article ID 453808, 9 pages, 2013.

[12] L. R. Nielsen and A. R. Kristensen, "Finding the $K$ best policies in a finite-horizon Markov decision process," European Journal of Operational Research, vol. 175, no. 2, pp. 1164-1179, 2006.

[13] J. Freixas and M. A. Puente, "Dimension of complete simple games with minimum," European Journal of Operational Research, vol. 188, no. 2, pp. 555-568, 2008.

[14] C. Bentz, D. Cornaz, and B. Ries, "Packing and covering with linear programming: a survey," European Journal of Operational Research, vol. 227, no. 3, pp. 409-422, 2013.

[15] A. Jiménez-Losada, J. R. Fernández, M. Ordóñez, and M. Grabisch, "Games on fuzzy communication structures with Choquet players," European Journal of Operational Research, vol. 207, no. 2, pp. 836-847, 2010.

[16] V. Voloshin and H.-J. Voss, "Circular mixed hypergraphs II: the upper chromatic number," Discrete Applied Mathematics, vol. 154, no. 8, pp. 1157-1172, 2006.

[17] J. Li, L. Wang, and H. Zhao, "On packing and coloring hyperedges in a cycle," Discrete Applied Mathematics, vol. 155, no. 16, pp. 2140-2151, 2007.

[18] P. D. Johnson Jr. and R. N. Mohapatra, "A class of inequalities relating degrees of adjacent nodes to the average degree in edge-weighted uniform hypergraphs," International Journal of Mathematics and Mathematical Sciences, vol. 2005, no. 21, pp. 3419-3426, 2005.

[19] D. Cheng and H. Qi, Semi-Tensor Product of Matrices: Theory and Applications, Science Press, Beijing, China, 2007.

[20] D. Cheng, H. Qi, and Z. Li, Analysis and Control of Boolean Networks: A Semi-Tensor Product Approach, Communications and Control Engineering Series, Springer, London, UK, 2011.

[21] D. Cheng and H. Qi, "Controllability and observability of Boolean control networks," Automatica, vol. 45, no. 7, pp. 16591667, 2009.

[22] D. Cheng and H. Qi, "A linear representation of dynamics of Boolean networks," IEEE Transactions on Automatic Control, vol. 55, no. 10, pp. 2251-2258, 2010.

[23] D. Cheng, Z. Li, and H. Qi, "Realization of Boolean control networks," Automatica, vol. 46, no. 1, pp. 62-69, 2010.

[24] D. Cheng, "Disturbance decoupling of boolean control networks," IEEE Transactions on Automatic Control, vol. 56, no. 1, pp. 2-10, 2011.

[25] D. Cheng, H. Qi, Z. Li, and J. B. Liu, "Stability and stabilization of Boolean networks," International Journal of Robust and Nonlinear Control, vol. 21, no. 2, pp. 134-156, 2011.

[26] D. Laschov and M. Margaliot, "A maximum principle for singleinput Boolean control networks," IEEE Transactions on Automatic Control, vol. 56, no. 4, pp. 913-917, 2011.

[27] F. Li and J. Sun, "Controllability of Boolean control networks with time delays in states," Automatica, vol. 47, no. 3, pp. 603607, 2011.

[28] H. Li and Y. Wang, "Boolean derivative calculation with application to fault detection of combinational circuits via the semi-tensor product method," Automatica, vol. 48, no. 4, pp. 688-693, 2012.

[29] Z. Liu and Y. Wang, "Disturbance decoupling of mix-valued logical networks via the semi-tensor product method," Automatica, vol. 48, no. 8, pp. 1839-1844, 2012.

[30] J. Feng, J. Yao, and P. Cui, "Singular Boolean networks: semitensor product approach," Science China Information Sciences, vol. 56, no. 11, Article ID 112203, pp. 1-14, 2013.

[31] Y. Wang, C. Zhang, and Z. Liu, "A matrix approach to graph maximum stable set and coloring problems with application to multi-agent systems," Automatica, vol. 48, no. 7, pp. 1227-1236, 2012.

[32] R. A. Horn and C. R. Johnson, Topics in Matrix Analysis, Cambridge University Press, Cambridge, Mass, USA, 1991.

[33] C. Berge, Graphs and Hypergraphs, North-Holland, Amsterdam, The Netherlands, 1973.

[34] Y. N. Sotskov, V. S. Tanaev, and F. Werner, "Scheduling problems and mixed graph colorings," Optimization, vol. 51, no. 3, pp. 597624, 2002.

[35] F. S. Al-Anzi, Y. N. Sotskov, A. Allahverdi, and G. V. Andreev, "Using mixed graph coloring to minimize total completion time in job shop scheduling," Applied Mathematics and Computation, vol. 182, no. 2, pp. 1137-1148, 2006. 


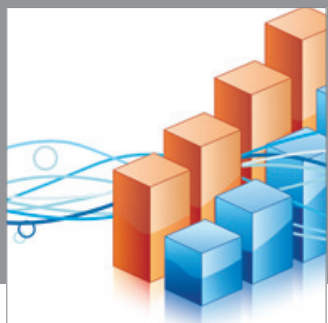

Advances in

Operations Research

mansans

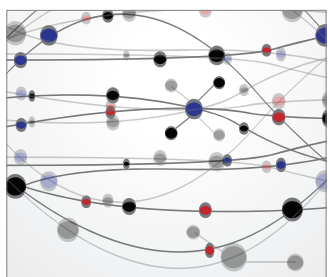

The Scientific World Journal
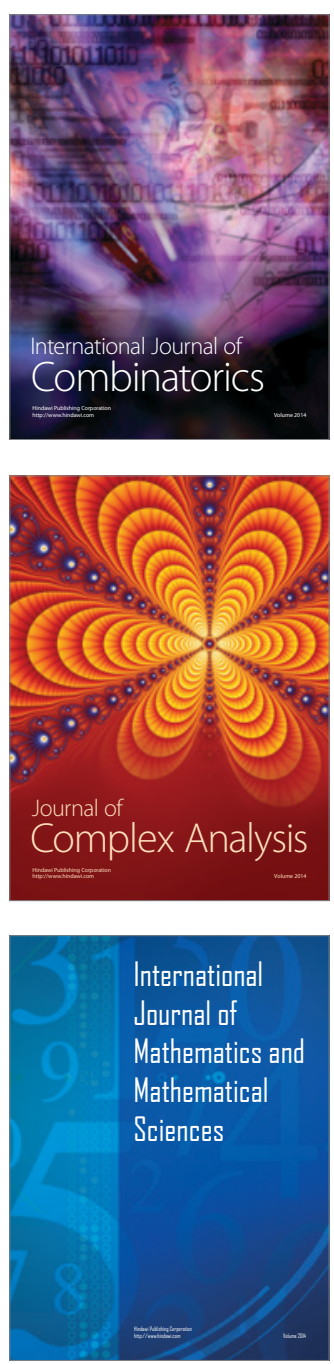
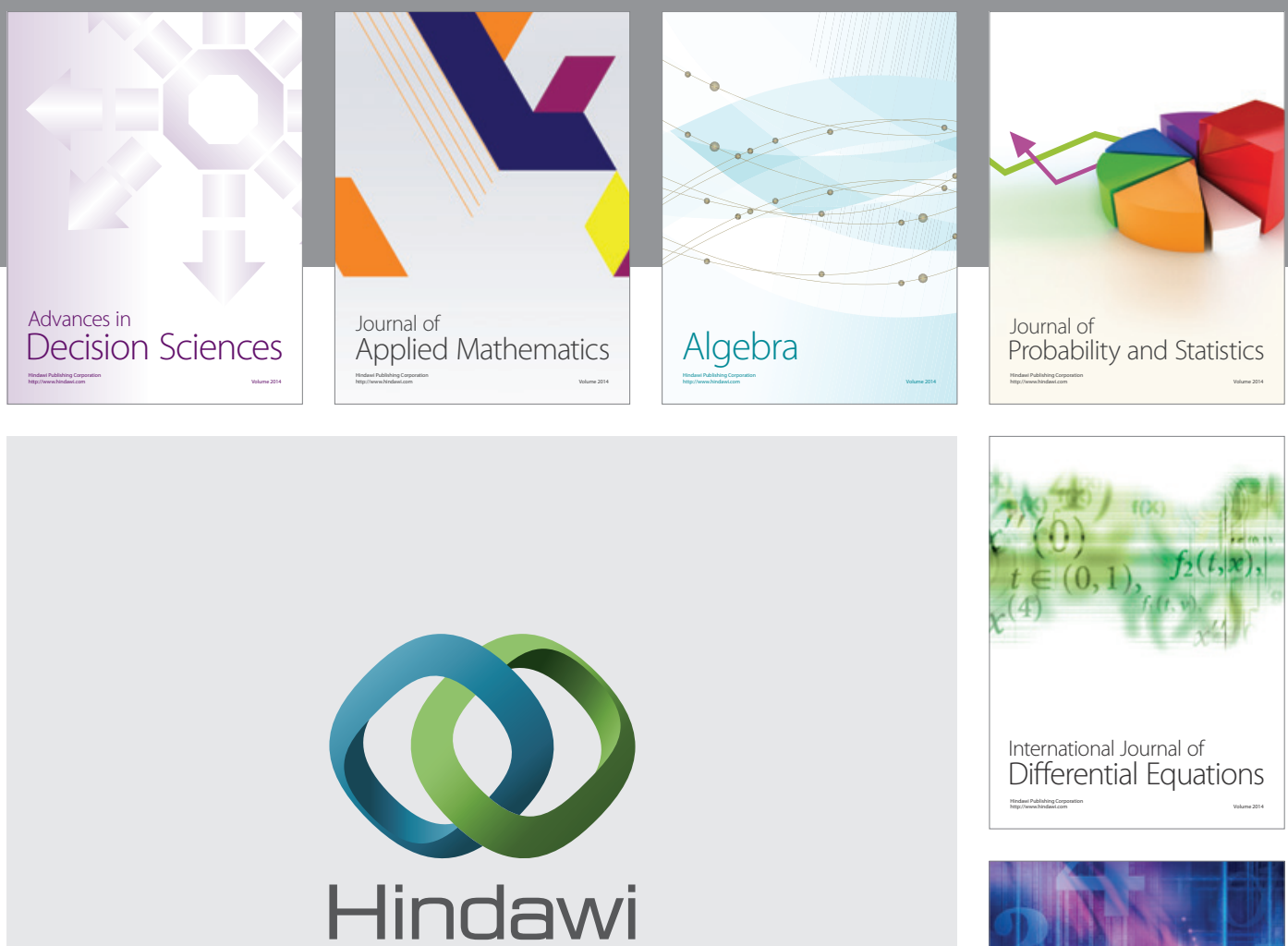

Submit your manuscripts at http://www.hindawi.com
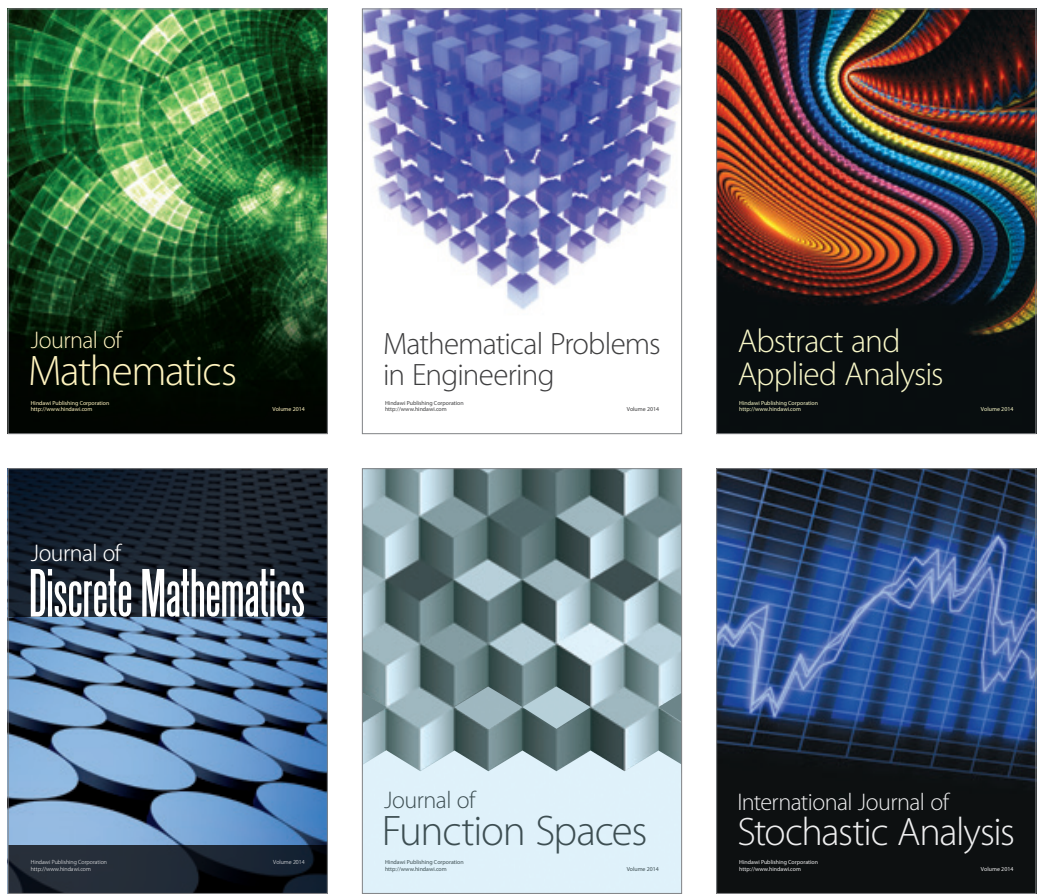

Journal of

Function Spaces

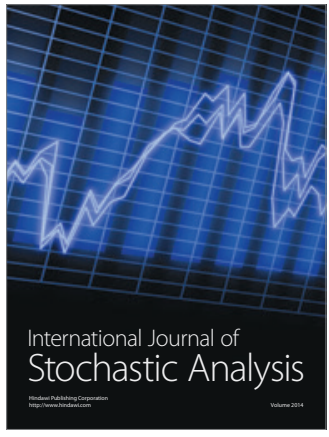

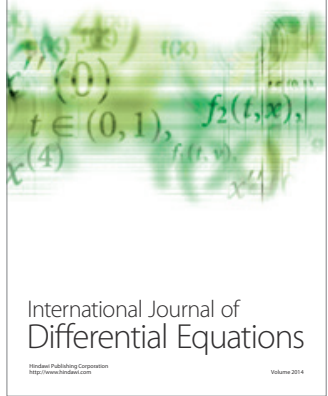
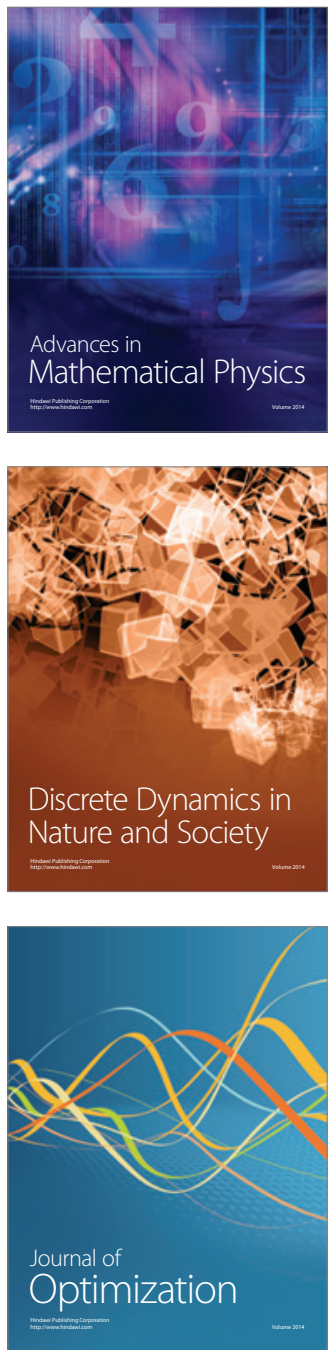